\title{
IDENTIFICATION OF NONLINEAR CASCADE SYSTEMS WITH TIME-VARYING BACKLASH
}

\author{
Jozef Vörös *
}

\begin{abstract}
Recursive identification of cascade systems with time-varying input backlash and linear dynamic system is presented. A new analytic form of backlash characteristic description is used, hence all the parameters in the cascade model equation are separated and their estimation is solved as a quasi-linear problem using the recursive least squares method with internal variable estimation. Simulation studies are included.
\end{abstract}

K e y w o r d s: nonlinear systems, backlash, cascade systems, recursive identification, time-varying systems

\section{INTRODUCTION}

One of the most important nonlinearities that limits control systems performance in many applications is the so-called backlash [19]. The backlash (see Fig. 1) can be classified as a hard (i.e. non-differentiable) and dynamic nonlinearity. It is well known that this kind of nonlinearity may often cause delays, oscillations and inaccuracy which severely limit the performance of control systems $[2,16,24,31-33]$. Therefore, compensation of backlash has attracted research effort by several decades [25, 27].

In most applications the backlash parameters are either poorly known or completely unknown, therefore the identification of backlash is fundamental for its compensation and implementation of corresponding inverse. Unfortunately, there are only few contributions in the literature on the identification of systems with hard nonlinearities $[1,30,34]$ and even fewer on backlash identification $[3,12,13,29,38]$. To the authors best knowledge, no contribution can be found which addresses the identification of cascade systems with time-varying input backlash.

In this paper a recursive identification method for cascade systems consisting of an input backlash followed by a linear dynamic system is presented. This is based on a new mathematical model for the backlash where this multi-valued nonlinearity is expressed in a special analytical form [37]. Application of a decomposition technique [34] leads to a cascade system description, which is quasilinear, and the parameters can be estimated using the recursive least squares (RLS) method with internal variable estimation based on available measured inputs and outputs [36]. The recursive method enables the on-line estimation of not only the parameters of linear dynamic system transfer function, but also the slopes of backlash and the constants determining the dead-zones, characterizing the time-varying nonlinear dynamic block, ie the approach deals with the problem never solved before. Simulation studies illustrate the feasibility of proposed identification method.

\section{BACKLASH}

The backlash characteristic with input $u(t)$ and output $x(t)$ is described by two straight lines, upward (right) and downward (left) sides of backlash, connected with horizontal line segments [3]. The backlash nonlinearity is shown in Fig. 1, and the mathematical model for the discrete-time case is given by

$$
x(t)= \begin{cases}m_{L}\left[u(t)+c_{L}\right], & u(t) \leq z_{L} \\ m_{R}\left[u(t)-c_{R}\right], & u(t) \geq z_{R} \\ x(t-1), & z_{L} \leq z_{R}\end{cases}
$$

where $m_{L}, m_{R}, c_{L}>0, c_{R}>0$ are constant parameters characterizing the backlash and

$$
\begin{aligned}
& z_{L}=\frac{x(t-1)}{m_{L}}-c_{L}, \\
& z_{R}=\frac{x(t-1)}{m_{R}}+c_{R}
\end{aligned}
$$

are the $u$-axis values of intersections of the two lines, with slopes $m_{L}, m_{R}$, with the horizontal inner segment containing $x(t-1)$. Evidently, the backlash is a first-order nonlinear dynamic system and (1) is not appropriate for the estimation of backlash parameters.

To describe the multi-valued nonlinearity (1) by one equation, a switching function and its complement are needed. One way, how to simplify the backlash description is the use of switching function [35]

$$
h(s)= \begin{cases}0, & \text { if } s>0 \\ 1, & \text { if } s \leq 0\end{cases}
$$

switching between two sets of values, ie, $(-\infty, s)$ and $(s, \infty)$. The complementary function to $h(s)$ is simply

* Slovak Technical University, Faculty of Electrical Engineering and Information Technology, Institute of Control and Industrial Informatics, Ilkovičova 3, 81219 Bratislava, Slovakia, jvoros@elf.stuba.sk 


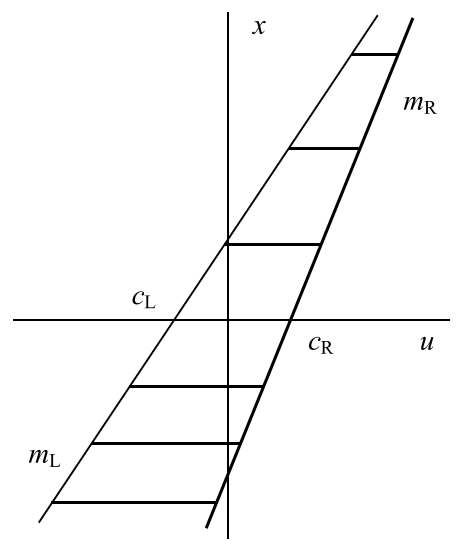

Fig. 1. Backlash characteristic

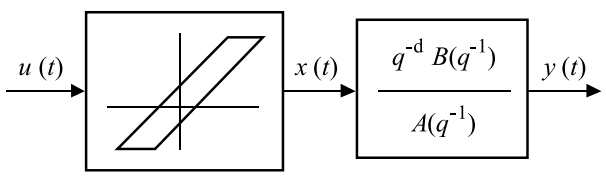

Fig. 2. Cascade system with backlash

$[1-h(s)]$. Now the following variables based on (4) can be defined

$$
\begin{aligned}
f_{1}(t) & =h\left[u(t)-z_{L}\right] \\
& =h\left\{\left[m_{L} u(t)+m_{L} c_{L}-x(t-1)\right] / m_{L}\right\}, \\
f_{2}(t) & =h\left[z_{R}-u(t)\right] \\
& =h\left\{\left[x(t-1)-m_{R} u(t)+m_{R} c_{R}\right] / m_{R}\right\}
\end{aligned}
$$

and the backlash can be modeled by one equation as

$$
\begin{array}{r}
x(t)=m_{L} u(t) f_{1}(t)+m_{L} c_{L} f_{1}(t)+m_{R} u(t) f_{2}(t)- \\
m_{R} c_{R} f_{2}(t)+x(t-1)\left[1-f_{1}(t)\right]\left[1-f_{2}(t)\right] .
\end{array}
$$

The input/output relation (7) is identical with that of (1). This backlash model allows the upward and downward line slopes to be different provided that the intersection of the two lines is not in the region of practical interest [37].

\section{CASCADE SYSTEMS WITH INPUT BACKLASH}

Cascade systems consist of serially connected linear and nonlinear subsystems. One of the simpliest cases is the connection of a static nonlinear subsystem followed by a linear dynamic one. This cascade system is known as the Hammerstein system and there are lots of identification methods for different types of nonlinearities and corresponding models, eg, $[4,5,7-$ $11,14,15,17,18,20,21,23,26,28,39]$.

In many real control systems the backlash appears in a cascade connection with a linear dynamic system. One of the possible cases is the cascade system where the backlash is followed by a linear dynamic system as shown in Fig. 2. The input nonlinearity is static in the Hammerstein system, while in the presented case the input nonlinearity is dynamic. It means that the cascase system consists of a nonlinear dynamic and a linear dynamic subsystem.

The linear dynamic system can be described by the difference equation model

$$
A\left(q^{-1}\right) y(t)=B\left(q^{-1}\right) x(t-d)
$$

where $x(t)$ and $y(t)$ are the inputs and outputs, respectively, $A\left(q^{-1}\right)$ and $B\left(q^{-1}\right)$ are scalar polynomials in the unit delay operator $q^{-1}$

$$
\begin{aligned}
& A\left(q^{-1}\right)=1+a_{1} q^{-1}+\cdots+a_{r} q^{-r} \\
& B\left(q^{-1}\right)=b_{0}+b_{1} q^{-1}+\cdots+b_{s} q^{-s}
\end{aligned}
$$

and $d>0$ is the pure delay. It is assumed that the constants $d, r$ and $s$ are known.

Assuming $b_{0}=1$ (one parameter can always be fixed in this model), the internal variable $x(\cdot)$ can be separated in the linear block description as follows

$$
y(t)=x(t-d)+\sum_{i=1}^{s} b_{i} x(t-d-i)-\sum_{j=1}^{r} y(t-j),
$$

and substitution of (7) only for the separated variable $\mathrm{x}(\mathrm{t}-\mathrm{d})$ leads to the following equation

$$
\begin{array}{r}
y(t)=m_{L} u(t-d) f_{1}(t-d)+m_{L} c_{L} f_{1}(t-d)+ \\
m_{R} u(t-d) f_{2}(t-d)-m_{R} c_{R} f_{2}(t-d)+ \\
x(t-d-1)\left[1-f_{1}(t-d)\right]\left[1-f_{2}(t-d)\right]+ \\
\sum_{i=1}^{s} b_{i} x(t-d-i)-\sum_{j=1}^{r} a_{j} y(t-j) .
\end{array}
$$

Defining the parameter and data vectors

$$
\begin{gathered}
\theta=\left[m_{1}, c_{1}, m_{2}, c_{2}, b_{1}, \ldots, b_{s}, a_{1}, \ldots, a_{r}\right]^{\top}, \\
m_{L}=m_{1}, c_{L}=\frac{c_{1}}{m_{1}}, m_{R}=m_{2}, c_{R}=\frac{c_{2}}{m_{2}},
\end{gathered}
$$

$$
\begin{gathered}
\varphi(t)=\left[u(t-d) f_{1}(t-d), f_{1}(t-d), u(t-d) f_{2}(t-d),\right. \\
-f_{2}(t-d), x(t-d-1), \ldots, x(t-d-s), \\
-y(t-1), \ldots,-y(t-r)]^{\top}
\end{gathered}
$$

and the corrected output, similarly as in [18]

$$
y_{c}(t)=y(t)-x(t-d-1)\left[1-f_{1}(t-d)\right]\left[1-f_{2}(t-d)\right]
$$

the model output equation can be written in the concise form as

$$
y_{c}(t)=\varphi^{\top}(t) \theta
$$




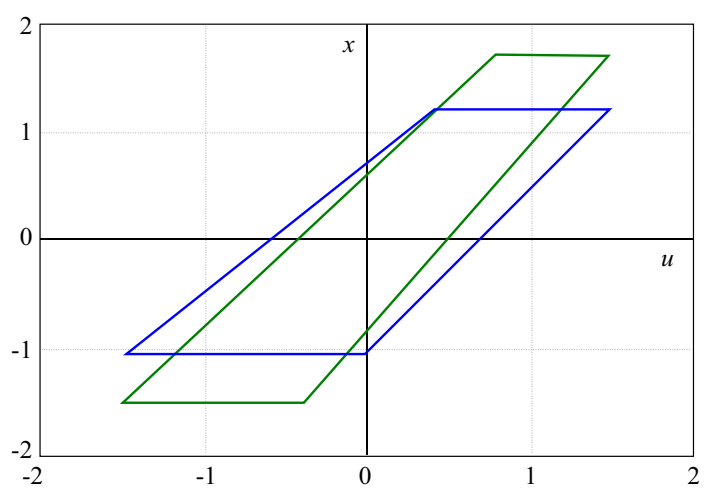

Fig. 3. Backlash characteristic - Example 1

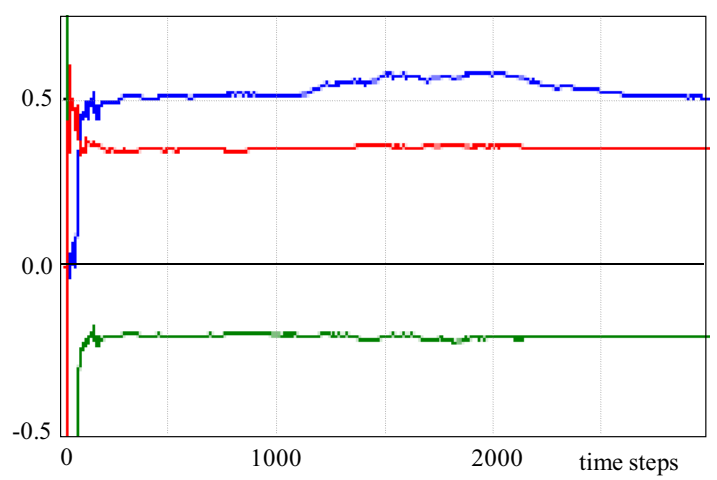

Fig. 5. Linear system parameter estimates — example 1

where the variables $f_{1}(t), f_{2}(t)$ and $x(t)$ depend on the vector $\theta$ and therefore (implicitly) $\varphi(t)=\varphi(t, \theta)$ and $y_{c}(t)=y_{c}(t, \theta)$.

The estimates of the parameter vector can be evaluated using the modified RLS algorithm, minimizing the quadratic criterion

$$
J(\theta, t)=\frac{1}{2} \sum_{k=1}^{t} \lambda^{t-k}\left[y_{c}(k)-\varphi^{\top}(k) \theta\right]^{2}
$$

where $0<\lambda \leq 1$ is the forgetting factor, the data vector $\varphi(t)$ and the corrected output $y_{c}(t)$ are replaced by $\hat{\varphi}(t)$ and $\hat{y}(t)$, respectively, with the estimates of corresponding internal variables. The recursive identification algorithm [22] supplemented with the internal variables estimation can be written as follows

$$
\begin{aligned}
\hat{\theta}(t) & =\hat{\theta}(t-1) \\
& +\frac{\hat{P}(t-1) \hat{\varphi}(t)\left[\hat{y}_{c}(t)-\hat{\varphi}^{\top}(t) \hat{\theta}(t-1)\right]}{\lambda+\hat{\varphi}^{\top}(t) \hat{P}(t-1) \hat{\varphi}(t)}, \\
\hat{P}(t) & =\frac{1}{\lambda}[\hat{P}(t-1) \\
& \left.-\frac{\hat{P}(t-1) \hat{\varphi}(t) \hat{\varphi}^{\top}(t) \hat{P}(t-1)}{\lambda+\hat{\varphi}^{\top}(t) \hat{P}(t-1) \hat{\varphi}(t)}\right], \\
\hat{f}_{1}(t-d) & =h\left\{\left[\hat{m}_{1}(t) u(t-d)\right.\right. \\
& \left.\left.+\hat{c}_{1}(t)-\hat{x}(t-d-1)\right] / \hat{m}_{1}(t)\right\}
\end{aligned}
$$

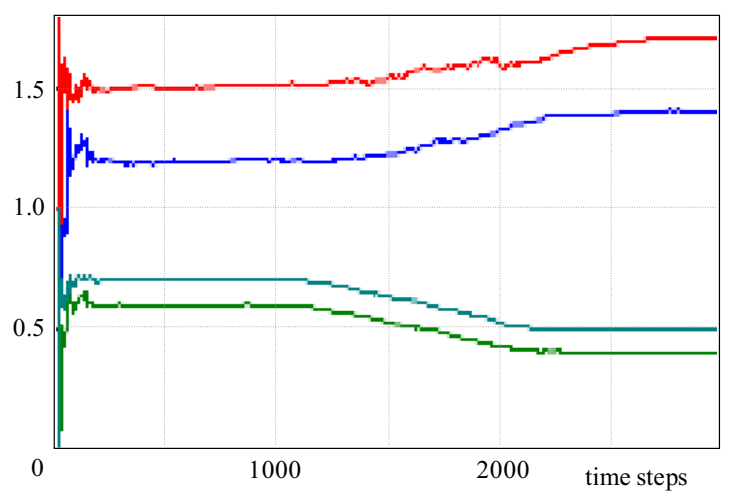

Fig. 4. Time-varying backlash parameter estimates — Example 1

$$
\begin{aligned}
& \hat{f}_{2}(t-d)=h\left\{\left[\hat{x}(t-d-1)-\hat{m}_{2}(t) u(t-d)\right.\right. \\
& \left.\left.+\hat{c}_{2}(t)\right] / \hat{m}_{2}(t)\right\} \\
& \hat{x}(t-d)=\hat{m}_{1}(t) u(t-d) \hat{f}_{1}(t-d)+\hat{c}_{1}(t) \hat{f}_{1}(t-d) \\
& +\hat{m}_{2} u(t-d) \hat{f}_{2}(t-d)-\hat{c}_{2} \hat{f}_{2}(t-d) \\
& +\hat{x}(t-d-1)\left[1-\hat{f}_{1}(t-d)\right]\left[1-\hat{f}_{2}(t-d)\right], \\
& \hat{\varphi}(t)=\left[u(t-d) \hat{f}_{1}(t-d), \hat{f}_{1}(t-d), u(t-d) \hat{f}_{2}(t-d),\right. \\
& -\hat{f}_{2}(t-d), \hat{x}(t-d-1), \ldots, \hat{x}(t-d-s), \\
& -y(t-1), \ldots,-y(t-r)]^{\top}, \\
& \hat{y}_{c}=y(t)-\hat{x}(t-d-1)\left[1-\hat{f}_{1}(t-d)\right]\left[1-\hat{f}_{2}(t-d)\right] \text {, } \\
& \hat{P}(0)=\mu I, \quad 0<\mu<\infty
\end{aligned}
$$

where the new values (estimates) of all the internal variables for the data vector and the corrected output in each recursion are computed by (21-25) with the previous estimates of corresponding parameters.

A bad initialization of a recursive algorithm leads generally to various problems such as convergence to a local minimum, to a wrong estimate or instability. For the proposed form of cascade model, the first estimates of backlash parameters must be chosen nonzero enabling to compute the first estimates of internal variables $f_{1}(t)$, $f_{2}(t)$ and $x(t)$ and the value of corrected output $y_{c}(t)$. It means that the initial values of $c_{L}$ and $c_{R}$ must be chosen as small positive real numbers, while the choice of the initial values of $m_{L}$ and $m_{R}$ has only one restriction, $i e$, the sign of initial estimates of the slopes of linear segments coincides with that of identified backlash. The input amplitudes must be large enough not to fall fully into the backlash deadzone area, however, they should not exceed the intersection of the upward and downward lines.

The key properties of the proposed algorithm (convergency, bias, consistency, etc) can be considered as analogous to those of the applied well-known recursive leastsquares algorithm [22] because the segments of backlash 


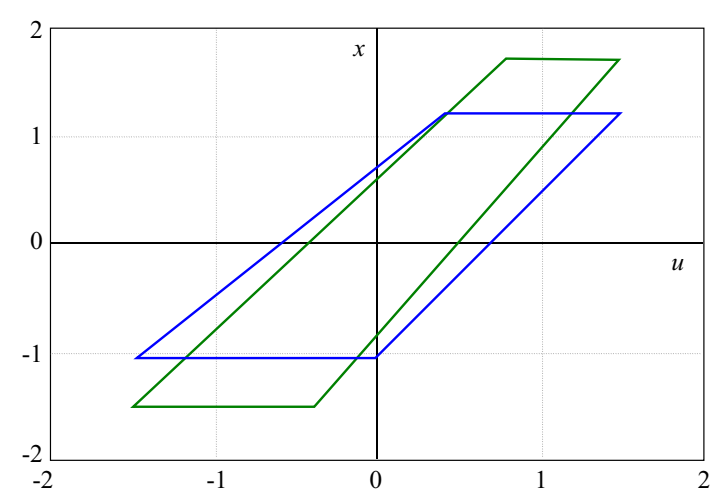

Fig. 6. Backlash characteristic - Example 2

are linear. The restriction is that only the estimates of internal variables are used in the data vector, which depend on the previous estimates of corresponding parameters.

\section{SIMULATION STUDIES}

The method for the recursive identification of systems with input backlash was implemented and tested in MATLAB. The performance of the proposed method is illustrated on examples of cascade systems with constant backlash parameters and cascade systems with timevarying backlash parameters.

ExAmple 1. This example shows the process of parameter estimation for a cascade system with time-varying backlash. The linear dynamic system was given by the difference equation

$y(t)=x(t-1)+0.5 x(t-2)+0.2 y(t-1)-0.35 y(t-2)$.

The nonlinear block was given by the original backlash characteristic (the thin line in Fig. 3) that was changed during the process to the new one (the thick line in Fig. 3). The corresponding sets of parameters are given in Table 1 .

Table 1. Backlash parameters — Example 1

\begin{tabular}{ccc}
\hline Parameter & Original & Changed \\
\hline$m_{L}$ & 1.2000 & 1.4000 \\
$c_{L}$ & 0.6000 & 0.4000 \\
$m_{R}$ & 1.5000 & 1.7000 \\
$c_{R}$ & 0.7000 & 0.5000 \\
\hline
\end{tabular}

The recursive identification was carried out for 3000 samples of uniformly distributed random inputs with $|u(t)|<1.5$ and simulated outputs. Normally distributed random noise with zero mean and signal to noise ratio (the square root of the ratio of output and noise variances) $S N R=50$ was added to the outputs. The changes of backlash parameters occurred slowly and gradually in the time interval $t \in(1000,2000)$. The recursive estimation algorithm was applied with initial values

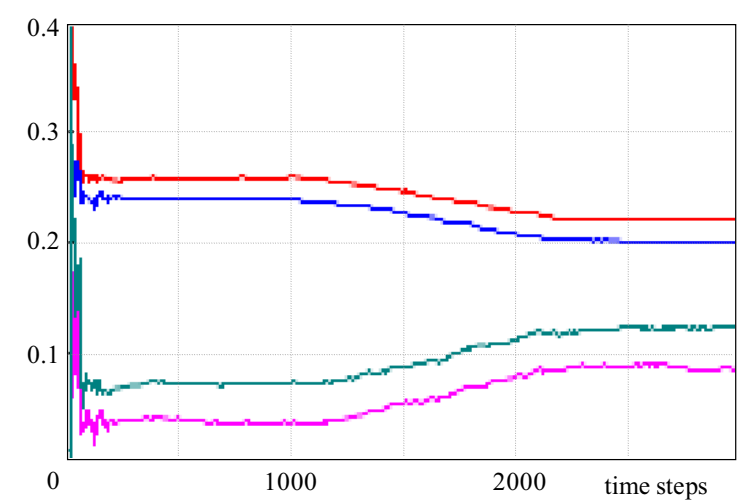

Fig. 7. Time-varying backlash parameter estimates - Example 2

$m_{L}=m_{R}=1$ and $c_{L}=c_{R}=0.001$ for the first estimates of $f_{1}(t), f_{2}(t)$ and $x(t)$, while the initial values of the linear system parameters were chosen zero. The use of a proper forgetting factor in the RLS is particularly useful in order to assign less weight to errors computed in the early steps of the procedure. However, as $\lambda$ decreases, noise sensitivity increases $[6,22]$. Therefore two forgetting factors were used in this example, ie $\lambda=0.9$ for the first 150 samples and $\lambda=0.995$ for the rest of data. The process of backlash parameter estimation is shown in Fig. 4 (the top-down order of parameters is $m_{R}, m_{L}, c_{R}, c_{L}$ ) and the estimates of linear block parameters are shown in Fig. 5 (the top-down order of parameters is $b_{1}, a_{2}$, $\left.a_{1}\right)$. It can be seen that the model parameter estimates are able to track the true parameters.

Example 2. The linear dynamic system in the cascade system with time-varying backlash was the same as in Example 1. The nonlinear block was given by the original backlash characteristic (the thin line in Fig. 6) that was changed during the process to the new one (the thick line in Fig. 6). The corresponding sets of parameters are given in Table 2.

Table 2. Backlash parameters - Example 2

\begin{tabular}{ccc}
\hline Parameter & Original & Changed \\
\hline$m_{L}$ & 0.2400 & 0.2000 \\
$c_{L}$ & 0.0350 & 0.0850 \\
$m_{R}$ & 0.2600 & 0.2200 \\
$c_{R}$ & 0.0700 & 0.1200 \\
\hline
\end{tabular}

The identification was performed on the basis of 3000 samples of uniformly distributed random inputs with $|u(t)|<1.0$ and simulated outputs. Normally distributed random noise with zero mean and $S N R=50$ was added to the outputs. The changes of backlash parameters occurred slowly and gradually in the time interval $t \in(1000,2000)$. However, the algorithm was applied with initial values $m_{L}=m_{R}=0.5$ and $c_{L}=c_{R}=0.01$ for the first estimates of $f_{1}(t), f_{2}(t)$ and $x(t)$, while the initial values of the linear system parameters were chosen zero. The process of backlash parameter estimation is 


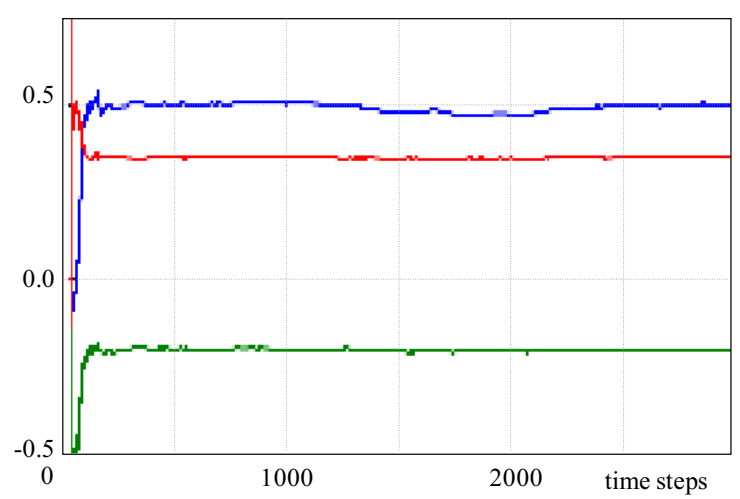

Fig. 8. Linear system parameter estimates — Example 2

shown in Fig. 7 (the top-down order of parameters is $m_{R}$, $\left.m_{L}, c_{R}, c_{L}\right)$ and the estimates of linear block parameters are shown in Fig. 8 (the top-down order of parameters is $\left.b_{1}, a_{2}, a_{1}\right)$.

The recursive algorithm proved good convergence in all the above cases. The parameter estimate fluctuations appearing in the early steps are not too severe. This is due to the fact that the segments of backlash are linear. The effects of the initialization of the proposed recursive algorithm on the quality of the estimates are almost marginal.

\section{CONCLUSIONS}

The identification of systems with unknown backlash is still an open theoretical problem of major relevance to control and other applications. The proposed identification method is a direct application of the known RLS algorithm [22] extended with the estimation of internal variables. It may be used in on-line monitoring and analysis of time-varying processes and also combined with on-line control strategies to produce adaptive control algorithms.

Although a convergence proof for the recursive identification of nonlinear systems with internal variable estimation is not available, the simulation studies have shown good convergence of the proposed algorithm. This is caused by the fact that the backlash has actually linear segments and the switching functions separate these segments. As always the corresponding linear segments of the nonlinearity are included into the computation, the convergence of estimates corresponds to that of the linear RLS method. Finally note, that the presented method can be easily extended for more complex cascaded systems with backlash.

\section{Acknowledgment}

The author gratefully acknowledges financial support from the Slovak Scientific Grant Agency (VEGA).

\section{REFERENCES}

[1] BAI, E. W.: Identification of Linear Systems with Hard Input Nonlinearities of Known Structure, Automatica 38 (2002), 853-860.

[2] BARREIRO, A.-BANOS, A. : Input-Output Stability of Systems with Backlash, Automatica 42 (2006), 1017-1024.

[3] CERONE, V.-REGRUTO, D. : Bounding the Parameters of Linear Systems with Input Backlash, IEEE Trans. Automatic Control 52 (2007), 531-536.

[4] CHEN, H. F.: Recursive System Identification, Acta Mathematica Scientia 29 B (2009), 650-672.

[5] CHEN, H. T.-HWANG, S. H.-CHANG, C. T. : Iterative Identification of Continuous-Time Hammerstein and Wiener Systems Using a Two-Stage Estimation Algorithm, Industrial \& Engineering Chemistry Research 48 (2009), 1495-1510.

[6] CHIDAMBARAM, M. : Computer Control of Processes, CRC Press, New York, 2001.

[7] DING, F.-CHEN, T. : Identification of Hammerstein Nonlinear ARMAX Systems, Automatica 41 (2005), 1479-1489.

[8] DING, F.-CHEN, T.-IWAI, Z. : Adaptive Digital Control of Hammerstein Nonlinear Systems with Limited Output Sampling, SIAM Journal on Control and Optimization 45 (2007), $2257-2276$

[9] DING, F.-SHI, Y.-CHEN, T. : Gradient-Based Identification Methods for Hammerstein Nonlinear ARMAX Models, Nonlinear Dynamics 45 (2006), 31-43.

10] DING, F.-SHI, Y.-CHEN, T. : Auxiliary Model-Based LeastSquares Identification Methods for Hammerstein Output-Error Systems, Systems \& Control Letters 56 (2007), 373-380.

[11] DOLANC, G.-STRMCNIK, S.: Identification of Nonlinear Systems Using a Piecewise-Linear Hammerstein Model, Systems \& Control Letters 54 (2005), 145-158.

[12] DONG, R.-TAN, Q.-TAN, Y.: Recursive Identification Algorithm for Dynamic Systems with Output Backlash and its Convergence, Int. J. Appl. Math. Comput. Sci. 19 (2009), 631-638.

13] GIRI, F.-ROCHDI, Y.-CHAOUI, F. Z.-BROURI, A. : Identification of Hammerstein Systems in Presence of Hysteresis-Backlash and Hysteresis-Relay Nonlinearities, Automatica 44 (2008), 767-775.

14] HASIEWICZ, Z.-MZYK, G.: Combined Parametric-Nonparametric Identification of Hammerstein Systems, IEEE Trans. on Automatic Control 49 (2004), 1370-1375.

15] HASIEWICZ, Z.-PAWLAK, M.-SLIWINSKI, P. : Nonparametric Identification of Nonlinearities in Block-Oriented Systems by Orthogonal Wavelets with Compact Support, IEEE Trans. Circuits and Systems I 52 (2005), 427-442.

[16] HÄGGLUND, T. : Automatic On-Line Estimation of Backlash in Control Loops, Journal of Process Control 17 (2007), 489-499.

17] JANCZAK, A. : Neural Network Approach for Identification of Hammerstein Systems, Int. J. Control 76 (2003), 1749-1766.

[18] JANCZAK, A. : Identification of Nonlinear Systems Using Neural Networks and Polynomial Models: A Block-Oriented Approach, Lecture Notes in Control and Information Sciences, Vol. 310, Springer-Verlag, Heidelberg, Berlin, 2005.

19] KALAŠ, V.-JURIŠICA, L.- ̌̌ALMAN, M.-ALMÁSSY, S.SIVIČEK, P.-VARGA, A.-KALAS̆, D. : Nonlinear and Numerical Servosystems, Alfa/SNTL, Bratislava, 1985. (in Slovak)

[20] LACY, S. L.-BERNSTEIN, D. S. : Subspace Identification for Non-Linear Systems with Measured-Input Non-Linearities, Int. J. Control 78 (2005), 906-926.

[21] LIU, Y.-BAI, E. W.: Iterative Identification of Hammerstein Systems, Automatica 43 (2007), 346-354.

[22] LJUNG, L.-SÖDERSTRÖM, T. : Theory and Practice of Recursive Identification, MIT Press, Cambridge, MA, 1983. 
[23] MZYK, G.: Generalized Kernel Regression Estimate for the Identification of Hammerstein Systems, Int. J. Applied Mathematics and Computer Science 17 (2007), 189-197.

[24] NORDIN, M.-GALIC, J.-GUTMAN, P. O. : New Models for Backlash and Gear Play, Int. J. Adapt. Control Signal Processing 11 (1997), 49-63.

[25] NORDIN, M.-GUTMAN, P. O.: Controlling Mechanical Systems with Backlash - a Survey, Automatica 38 (2002), 1633-1649.

[26] PUPEIKIS, R. : On the Identification of Hammerstein Systems Having Saturation-Like Functions with Positive Slopes, Informatica 17 (2006), 55-68.

[27] SELMIC, R. R.-LEWIS, F. L.: Neural Net Backlash Compensation with Hebbian Tuning Using Dynamic Inversion, Automatica 37 (2001), 1269-1277.

[28] SLIWINSKI, P.-ROZENBLIT, J.-MARCELLIN, M. W.KLEMPOUS, R.: Wavelet Amendment of Polynomial Models in Hammerstein Systems Identification, IEEE Trans. Automatic Control 54 (2009), 820-825.

[29] SUN, L.-LIU, W.-SANO, A. : Identification of a Dynamical System with Input Nonlinearity, IEE Proceedings - Control Theory Applications 146 (1999), 41-51.

[30] TAO, G.-CANUDAS de WIT, C., (Eds.): : Special Issue on Adaptive Systems with Non-Smooth Nonlinearities, Int. J. Adapt. Control Signal Processing 11 (1997).

[31] TAO, G.-KOKOTOVIC, P. V.: Adaptive Control of Systems with Backlash, Automatica 29 (1993), 323-335.

[32] TAO, G.-KOKOTOVIC, P. V.: Adaptive Control of Systems with Unknown Output Backlash, IEEE Trans. Automatic Control 40 (1995), 326-330.

[33] TAO, G.-KOKOTOVIC, P. V.: Adaptive Control of Systems with Unknown Non-Smooth Non-Linearities, Int. J. Adapt. Control Signal Processing 11 (1997), 81-100.
34] VÖRÖS, J. : Parameter Identification of Discontinuous Hammerstein Systems, Automatica 33 (1997), 1141-1146.

35] VÖRÖS, J.: Modeling and Parameter Identification of Systems with Multisegment Piecewise-Linear Characteristics, IEEE Trans. Automatic Control 47 (2002), 184-188.

[36] VÖRÖS, J.: Recursive Identification of Hammerstein Systems with Discontinuous Nonlinearities Containing Dead-Zones, IEEE Trans. Automatic Control 48 (2003), 2203-2206.

[37] VÖRÖS, J. : Modeling and Identification of Systems with Backlash, Automatica 46 (2010), 369-374.

[38] WANG, J.-SANO, A.-CHEN, T.-HUANG, B. : Identification of Hammerstein Systems without Explicit Parameterization of Nonlinearity, Int. J. Control 82 (2009), 937-952.

[39] ZHANG, X.-TAN, Y.: Modelling of Ultrasonic Motor with Dead-Zone Based on Hammerstein Model Structure, Journal of Zhejiang University - Science A 9 (2008), 58-64.

Received 19 March 2010

Jozef Vörös (Ing, PhD) was born in Hurbanovo on July 9, 1949. He graduated in automatic control from the Faculty of Electrical Engineering of Slovak Technical University, Bratislava in 1974 and received his PhD degree in control theory from the Institute of Technical Cybernetics of the Slovak Academy of Sciences, Bratislava in 1983. Since 1992 he has been with the Faculty of Electrical Engineering and Information Technology at the Slovak Technical University in Bratislava where he is acting as a senior research scientist in the Institute of Control and Industrial Informatics. His research interests include the analysis and identification of nonlinear systems. He is also interested in the area of mobile robot path planning using quadtree and octree representations.

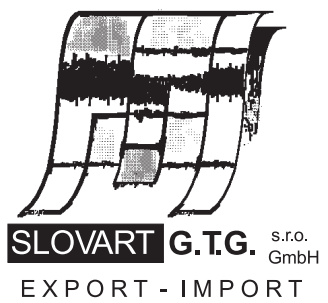

EXPORT - IMPORT
EXPORT - IMPORT

of periodicals and of non-periodically printed matters, books and CD-ROMs

Krupinská 4 PO BOX 152, 85299 Bratislava 5, Slovakia tel: ++421 263839 472-3, fax: ++421263839485 info@slovart-gtg.sk; http://www.slovart-gtg.sk

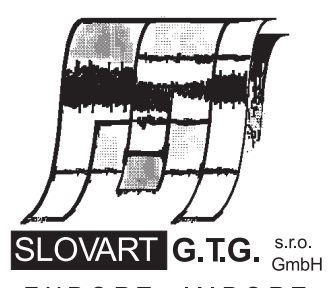

EXPORT - IMPORT 